\title{
Back Extensor Strengthening Exercise and Backpack Wearing Treatment for Camptocormia in Parkinson's Disease: A Retrospective Pilot Study
}

\author{
Kun Hee Lee, MD, Jong Moon Kim, MD, Hyoung Seop Kim, MD
}

Department of Physical Medicine and Rehabilitation, National Health Insurance Service Ilsan Hospital, Goyang, Korea

\begin{abstract}
Objective To investigate the effect of a conservative treatment regime in Parkinson's disease patients with camptocormia.

Methods Nine patients with Parkinson's disease were included in a retrospective pilot study of the value of back extensor strengthening exercise. Six inpatients received a 30-minute treatment, twice daily for 5 weeks, being treated on average for 34 days; while three outpatients visited the clinic and were educated for home exercise and backpack wearing treatment. Outpatients should be scheduled to visit the outpatient department to check physical status every 2-4 weeks for an average of 3 months.

Results All patients except one showed statistically significant improvements in activities of daily living (ADL) and motor symptoms, as measured by flexion angle at standing posture, Unified Parkinson's Disease Rating Scale (UPDRS) II and III, and modified Hoehn-Yahr staging.

Conclusion Conservative treatment is effective in postural correction of camptocormia in Parkinson's disease, as well as improvement in ADL and motor symptoms.
\end{abstract}

Keywords Camptocormia, Parkinson disease, Rehabilitation, Physical therapy modalities

\section{INTRODUCTION}

Parkinson's disease is a neurological disorder that involves the degeneration of dopaminergic neurons in the substantia nigra. It is relatively common, affecting ap- proximately $1 \%$ of the population over 60 years of age, and is characterized by motor symptoms such as tremor, rigidity, bradykinesia and postural instability. Of these, postural instability such as camptocormia is one of the most common symptoms, observed in $3 \%-17 \%$ of all Par-

Received September 12, 2016; Accepted November 4, 2016

Corresponding author: Hyoung Seop Kim

Department of Physical Medicine and Rehabilitation, National Health Insurance Service Ilsan Hospital, 100 Ilsan-ro, Ilsandong-gu, Goyang 10444, Korea. Tel: +82-31-300-0137, Fax: +82-31-900-0343, E-mail: rehappydoc@gmail.com

ORCID: Kun Hee Lee (http://orcid.org/0000-0001-7904-9840); Jong Moon Kim (http://orcid.org/0000-0002-8684-8736); Hyoung Seop Kim (http:// orcid.org/0000-0002-5310-4802).

(c) This is an open-access article distributed under the terms of the Creative Commons Attribution Non-Commercial License (http://creativecommons.org/ licenses/by-nc/4.0) which permits unrestricted noncommercial use, distribution, and reproduction in any medium, provided the original work is properly cited. Copyright (c) 2017 by Korean Academy of Rehabilitation Medicine 
kinson's disease patients [1-8].

Camptocormia is an axial postural deformity characterized by abnormal spinal flexion. It differs from kyphosis in that the kyphotic deformity can be corrected by lying down in a recumbent position, use of a high frame walker, or standing against a wall [3]. The pathophysiology of camptocormia is not defined yet, but several hypotheses have been proposed; it may be a symptom of Parkinson's disease progression, a form of dystonia in the course of Parkinson's disease, a result of paraspinal myopathy that either follows or coincides with Parkinson's disease, or a side effect of the medications used in Parkinson's disease [4]. In addition, spinal deformity of this kind is difficult to distinguish from other axial skeletal disorders that are common in the elderly. Currently, there is no gold standard treatment available, with only a few case reports of pharmacological, surgical, and conservative treatment options. Conservative options include plaster corset, lowslung backpack, high-frame walker with forearm support, thoraco-pelvic anterior distraction orthosis, taping, and physiotherapy-treatments. Previously, we reported a case study of effective correction of camptocormia by applying back strengthening and cruciform anterior spinal hyperextension brace [6]. In this study, we present a retrospective data collection and comparative analysis of clinical indices, in 9 cases of rehabilitation treatment, that comprised back extensor strengthening exercise, core muscle exercise, and back pack wearing in Parkinson's disease patients with camptocormia. Through this study, we aim to provide a rationale for further investigation and application of conservative treatment.

\section{MATERIALS AND METHODS}

Enrolled patients were diagnosed with Parkinson's disease by expert neurologists and referred to the department of physical medicine and rehabilitation at our clinic, for postural instability, between March 2013 and August 2015. Camptocormia was diagnosed by an expert physiatrist and was differentiated from kyphosis by lying down in a recumbent position. Most authors define camptocormia as thoracolumbar flexion at least $45^{\circ}$ when standing or walking. But this definition is known to be somewhat arbitrary and therefore we focused on limitation of ADL and motor symptoms [9]. As a result, we defined camptocormia as abnormal thoracolumbar flexion at least $5^{\circ}$, which resolves when the patient lies supine, and was accompanied by limitations of ADL and motor symptoms.

Nine patients ( 6 women and 3 men) included in the study had varying durations of Parkinson's disease and camptocormia. All patients were taking anti-Parkinson's medication, one patient had accompanying right pontine infarction, and three patients had a history of surgical treatment for spine deformity (Table 1). Dosage of antiParkinson's disease medication was constant during the follow-up period and deep brain stimulation (DBS) was not performed in all patients and freezing gait was not shown in all patients. We defined the duration of Parkinson's disease as time from diagnosis of Parkinson's disease and duration of camptocormia as time from abnormal thoracolumbar flexion. In cases \#1, \#2 and \#4, the duration of camptocormia was longer than the duration of Parkinson's disease. We examined for the presence of pathologic fracture or previous spinal operation history by X-ray evaluation before rehabilitation treatment.

The camptocormia flexion angles were measured by lateral view gross photograph [1] (Fig. 1). The three points used for measuring the flexion angle were midpoint of foot, crossing point between horizontal line from anterior superior iliac spine and vertical line from greater trochanter and midpoint of auricle. Flexion angle was measured after 5 minutes of free ambulation. UPDRS II, III and modified Hoehn-Yahr staging were recorded at the beginning and at the end of treatment for each patient (Table 2).

Six inpatients received a 30-minute treatment twice a day for 5 weeks, for an average of 34 days (range, 23-44 days). Treatment sessions were composed of 20 minutes of physiotherapy-core muscle strengthening exercise, pelvic tilting exercise, back extensor strengthening exercise and bending side muscle stretching exercise, more focused on strengthening (Fig. 2A-2C). Then 10 minutes of treatment comprised ambulation while wearing a lowslung backpack (Fig. 2D). The weight of the backpack started at $1.5 \mathrm{~kg}$. Depending on tolerability, we increased the weight of the backpack until camptocormia was corrected in a standing position. Patients were educated to wear the low-slung backpack at least 2 hours daily while ambulating. Anterior spinal hyperflexion brace was applied to 1 patient who was not able to tolerate the lowslung backpack due to its weight (Fig. 2E). 


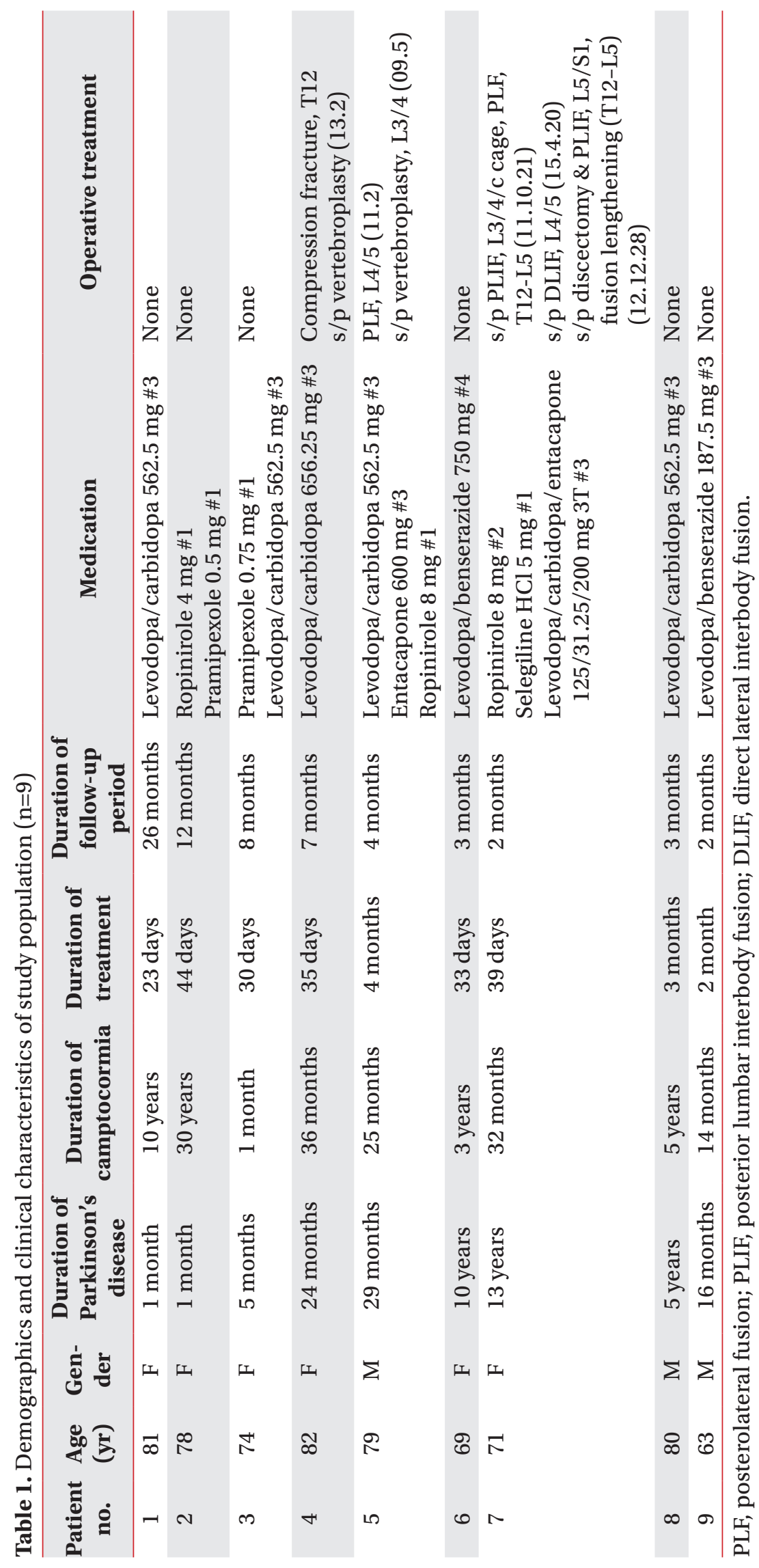



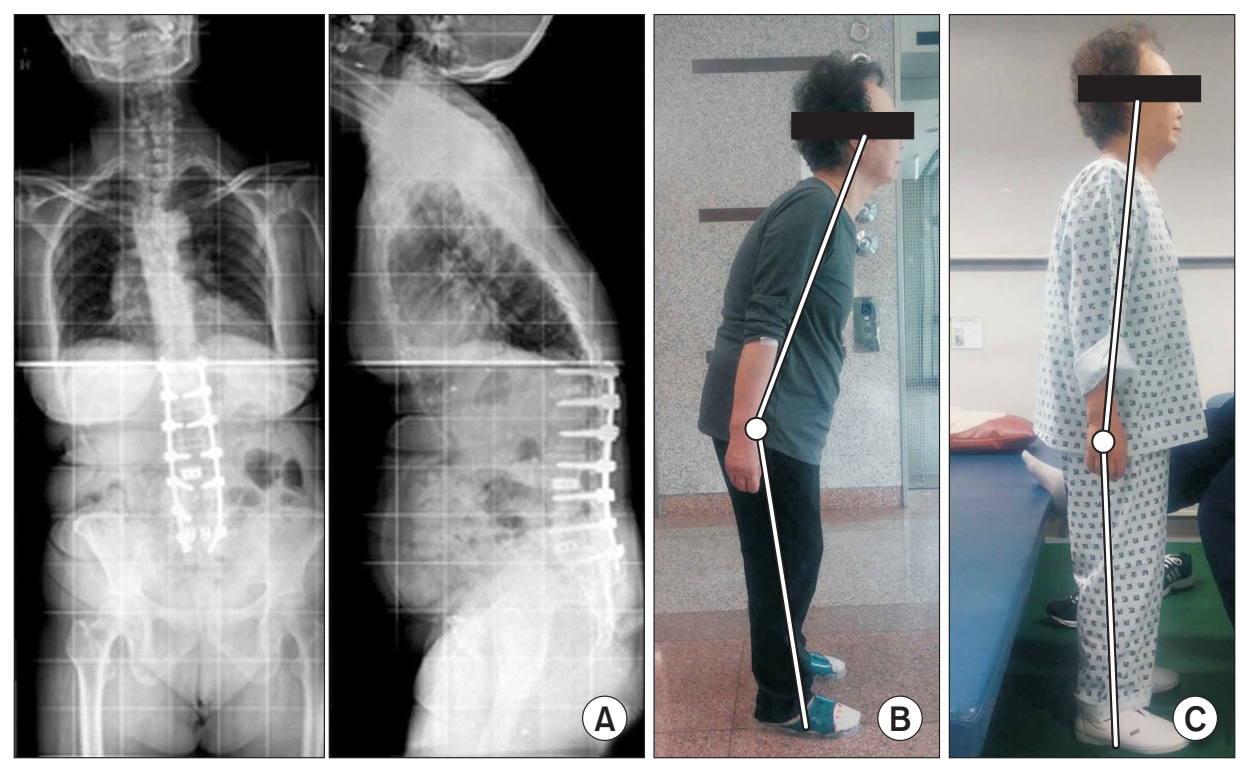

Fig. 1. Patient \#7 before and after treatment. (A) She had an operative treatment for kyphosis and lower back pain but showed camptocormia afterwards. (B) Lateral image before rehabilitation treatment. (C) Lateral image after rehabilitation treatment.

Table 2. Changes in angle, UPDRS, and modified Hoehn-Yahr staging after treatment

\begin{tabular}{|c|c|c|c|c|c|c|c|c|c|}
\hline $\begin{array}{c}\text { Patient } \\
\text { no. }\end{array}$ & $\begin{array}{l}\text { Treat- } \\
\text { ment }\end{array}$ & $\begin{array}{c}\text { Angle } \\
\left({ }^{\circ}\right)\end{array}$ & $\begin{array}{c}\Delta \text { Angle } \\
\left({ }^{\circ}\right)\end{array}$ & $\begin{array}{c}\text { UPDRS } \\
\text { II }\end{array}$ & $\begin{array}{c}\Delta \text { UPDRS } \\
\text { II }\end{array}$ & $\begin{array}{c}\text { UPDRS } \\
\text { III }\end{array}$ & $\begin{array}{c}\Delta \text { UPDRS } \\
\text { III }\end{array}$ & $\begin{array}{c}\text { Modified } \\
\text { Hoehn-Yahr } \\
\text { staging }\end{array}$ & $\begin{array}{c}\Delta \text { Modified } \\
\text { Hoehn-Yahr } \\
\text { staging }\end{array}$ \\
\hline \multirow[t]{2}{*}{1} & Pre & 43 & 0 & 17 & 0 & 27 & 0 & 3 & 0 \\
\hline & Post & 43 & & 17 & & 27 & & 3 & \\
\hline \multirow[t]{2}{*}{2} & Pre & 77 & 62 & 10 & 3 & 16 & 5 & 3 & 0.5 \\
\hline & Post & 15 & & 7 & & 11 & & 2.5 & \\
\hline \multirow[t]{2}{*}{3} & Pre & 20 & 18 & 12 & 2 & 21 & 5 & 3 & 0.5 \\
\hline & Post & 2 & & 10 & & 16 & & 2.5 & \\
\hline \multirow[t]{2}{*}{4} & Pre & 24 & 4 & 23 & 9 & 43 & 7 & 4 & 1.5 \\
\hline & Post & 20 & & 14 & & 36 & & 2.5 & \\
\hline \multirow[t]{2}{*}{5} & Pre & 41 & 9 & 15 & 2 & 32 & 4 & 3 & 0.5 \\
\hline & Post & 32 & & 13 & & 28 & & 2.5 & \\
\hline \multirow[t]{2}{*}{6} & Pre & 6 & 12 & 11 & 5 & 40 & 6 & 3 & 0.5 \\
\hline & Post & -6 & & 6 & & 34 & & 2.5 & \\
\hline \multirow[t]{2}{*}{7} & Pre & 24 & 19 & 6 & 1 & 20 & 2 & 2.5 & 0.5 \\
\hline & Post & 5 & & 5 & & 18 & & 2 & \\
\hline \multirow[t]{2}{*}{8} & Pre & 49 & 47 & 13 & 1 & 31 & 3 & 3 & 0.5 \\
\hline & Post & 2 & & 12 & & 28 & & 2.5 & \\
\hline \multirow[t]{2}{*}{9} & Pre & 12 & 15 & 13 & 2 & 27 & 2 & 3 & 0.5 \\
\hline & Post & -3 & & 11 & & 25 & & 2.5 & \\
\hline Mean & & \multicolumn{2}{|c|}{20.667} & \multicolumn{2}{|c|}{2.778} & \multicolumn{2}{|c|}{3.778} & \multicolumn{2}{|c|}{0.556} \\
\hline $\mathrm{p}$-value $\mathrm{e}^{\mathrm{a})}$ & & \multicolumn{2}{|c|}{$0.012^{*}$} & \multicolumn{2}{|c|}{$0.011^{*}$} & \multicolumn{2}{|c|}{$0.012^{*}$} & \multicolumn{2}{|c|}{$0.007^{* *}$} \\
\hline
\end{tabular}

UPDRS, Unified Parkinson's Disease Rating Scale.

${ }^{*} \mathrm{p}<0.05$ between pre- and post-treatment, ${ }^{* *} \mathrm{p}<0.01$ between pre- and post-treatment.

${ }^{a)}$ Wilcoxon signed-rank test. 

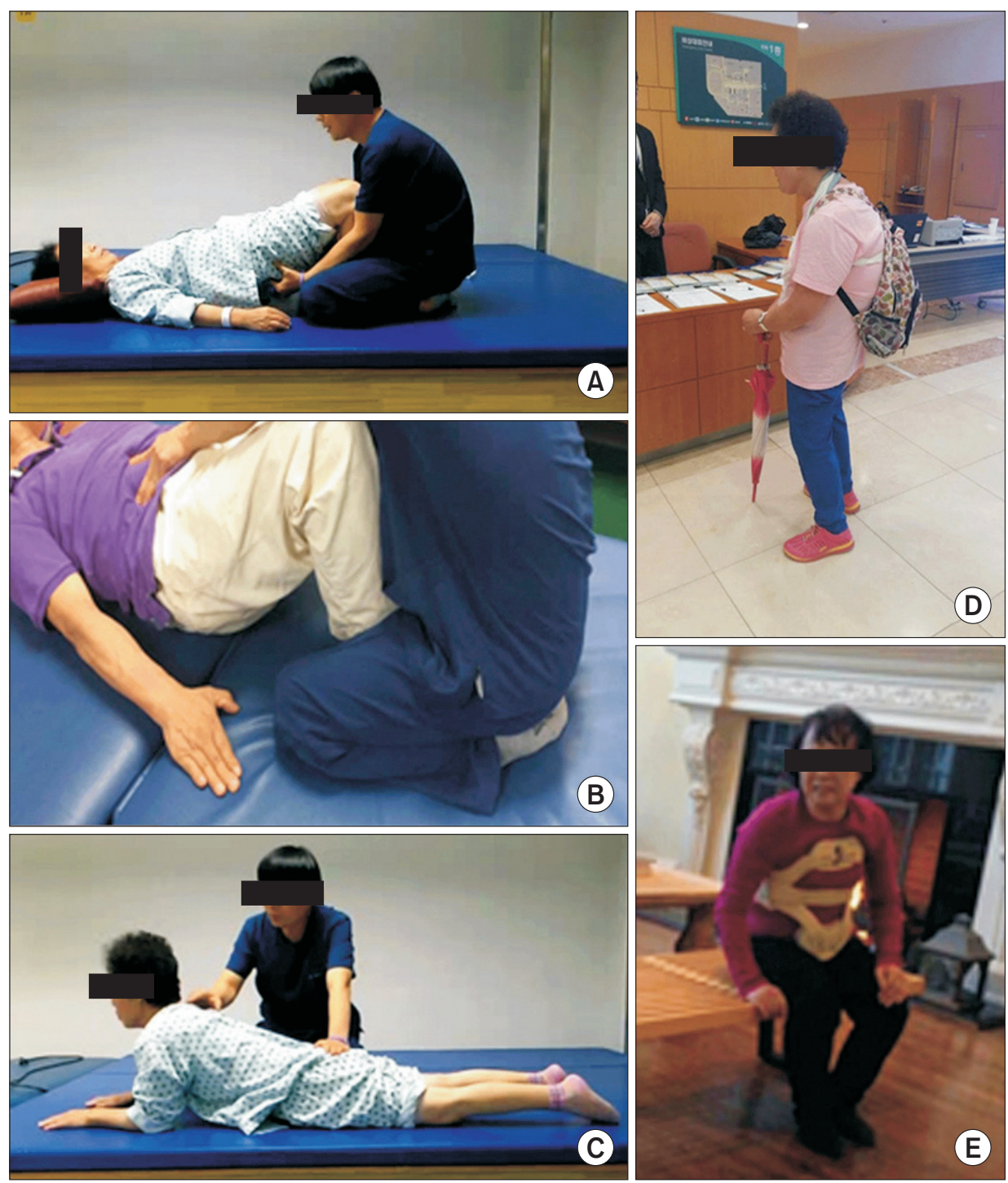

Fig. 2. Various modalities of conservative management for camptocormia. (A) Core muscle strengthening exercise. (B) Pelvic tilting exercise. (C) Back extensor strengthening exercise. (D) Lowslung backpack. (E) Anterior spinal hyperflexion brace.
Three outpatients received treatment once a week for an average of 3 months, to undergo back extensor strengthening exercise as well as backpack wearing treatment focusing on education for home exercise. The composition of treatment for these patients was not very different from the inpatients. Moreover, should be scheduled to visit the outpatient department to check physical status every 2-4 weeks for evaluation of compliance and determining the weight of the low-slung backpack.

All data were collected from medical charts record post-treatment. Clinical improvement was assessed by the change between pre- and post- rehabilitation treatment flexion angle in the standing position ( $\Delta$ angle), UPDRS ( $\triangle$ UPDRS), and modifided Hoenh-Yahr staging ( $\Delta$ modifided Hoenh-Yahr staging). For statistical analysis
Wilcoxon rank sum test was used and analyzed by SPSS software ver. 22.0 for Windows (IBM, Armonk, NY, USA).

\section{RESULTS}

Camptocormia was improved in all patients but one (case \#1) in angle, UPDRS II and III, and modified HoehnYahr staging (Fig. 3). The most dramatic improvements were observed in case $\# 2$ for angle $\left(\triangle\right.$ angle $\left.=62^{\circ}\right)$, and case \#4 for UPDRS II ( $\triangle$ UPRS II=9), UPDRS III ( $\triangle$ UPDRS III=7), and modified Hoehn-Yahr staging ( $\Delta$ modified HoehnYahr staging=1.5). In case \#8, camptocormia initially came back with impaired walking when the lower slung backpack was removed, but at 3 months post-treatment all patients except case \#1 showed improved spine angle. 


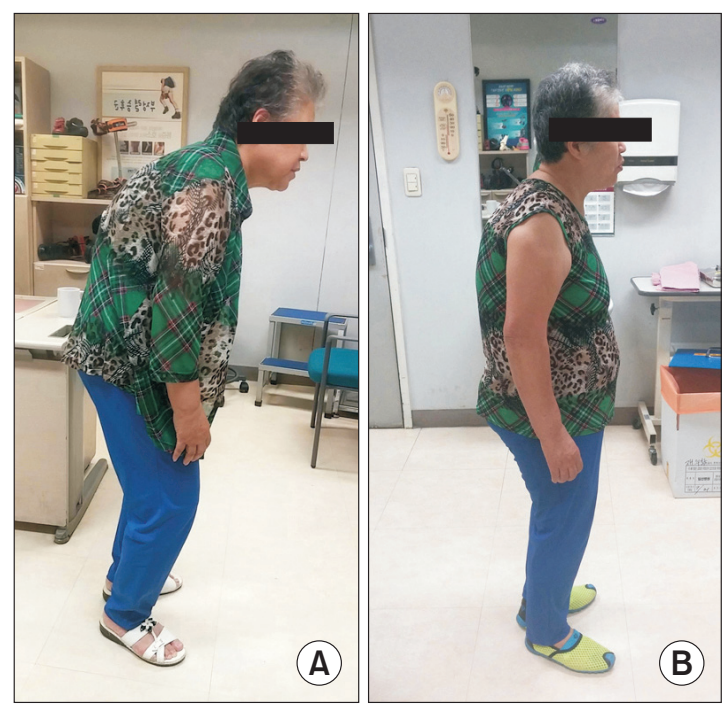

Fig. 3. Patient \#4 before and after treatment. She was educated for home exercise at the outpatient clinic. After 4 weeks of home exercise and low-slung backpack treatment, she showed improvement of camptocormia. (A) Lateral image before rehabilitation treatment. (B) Lateral image after rehabilitation treatment.

Therapeutic exercise was effective in improving angle (mean $\Delta$ angle $=20.667^{\circ}$ ), ADL (mean $\Delta$ UPRS II $=2.778$ ), and motor symptom index (mean $\Delta$ UPRS III=3.778; mean $\Delta$ modified Hoehn-Yahr staging=0.556) of Parkinson's disease patients with camptocormia. Statistical significance was verified by Wilcoxon signed-rank test $(\mathrm{p}<0.05)$ (Table 2).

Case \#4 patient showed a relatively small improvement in angle, but showed the largest improvement in UPDRS II \& III and modified Hoehn-Yahr staging, suggesting that ADL and motor symptom evaluation is more important that just the angle itself in respect to treatment of camptocormia in Parkinson's disease.

\section{DISCUSSION}

Based on different hypotheses regarding the pathogenesis of camptocormia, several treatment approaches have been suggested. The possible etiologies include the progression of Parkinson's disease, dyskinetic movement due to Parkinsonism medication, Parkinsonism with dystonia, and paraspinal myopathy.

For camptocormia associated with Parkinson's disease progression, high-dose dopaminergic medication such as levodopa has been used. However, in one study only $20 \%$ of the subjects responded to levodopa treatment [8], while another study reported that high-dose levodopa may result in dyskinetic movement in Parkinson's disease patients, suggesting that a reduced use of dopaminergics is recommended [2]. Some studies that have regarded camptocormia as a form of dystonia in Parkinson's disease have utilized botulinum toxin injection in the rectus abdominis and iliopsoas muscles, but the efficacy of this treatment remains controversial $[5,7,10]$.

Paraspinal myopathy that occurs due to or accompanying Parkinson's disease is another possible cause of camptocormia. In this regard, orthopedic surgical correction has been suggested as a possible treatment, while some beneficial effects of pallidotomy and deep brain stimulation have been reported. However, the efficacy of these treatments is still inconclusive [11-16]. An epidemiological and clinical study by Tiple et al. [3] proposed that a history of vertebral surgery was a risk factor for the pathogenesis of camptocormia, while Upadhyaya et al. [17] reported that spinal surgery in Parkinson's disease patients bears a high rate of postoperative complications, and that the presence of myelopathy or radiculopathy due to preoperative spinal stenosis must be considered.

Among conservative treatment reports, Gerton et al. [18] reported the first case study using backpack-wearing to reduce the degree of flexion, and Schroeteler et al. [19] reported three cases where high frame walker with forearm support was used to improve back pain and walking distance. On the other hand, De Seze et al. [20] performed thoraco-pelvic anterior distraction orthosis and physiotherapy on 15 patients, which resulted in increased lumbar lordosis, reduced pain, and enhanced quality of life, and Ye et al. [6] reported one case showing that a cruciform anterior spinal hyperflexion device and back extension strengthening exercise, resolved ambulatory disability and flexion posture permanently.

While most camptocormia treatment studies have been case reports, Capecci et al. [21] conducted a single-blind, randomized controlled trial with 20 Parkinsonism patients with postural deformity. In their study, 7 patients in a tailored postural rehabilitation group and 6 patients with tailored postural rehabilitation treatment and Kinesio taping, showed improved measures of trunk posture sagittal and coronal angles, gait and balance, in accordance with our study. Our study is somewhat different 
in that Capecci et al. [21] focused on tactile stimulation, stretching and postural re-education rather than back extensor strengthening exercise.

In the present study, our case \#1 patient did not show improvement in camptocormia after conservative treatment. Previously Asahi et al. [1] have reported that bilateral subthalamic deep brain stimulation was not effective in patients with paraspinal muscle degeneration, which was also found in case \#1 patient as determined by lumbar spine magnetic resonance imaging (Fig. 4). In addition, Schulz-Schaeffer et al. [22] found that myopathy develops when secondary changes such as intrafascicular fibrosis and fatty degeneration progresses over time, proposing a hypothesis that the timing of neurostimulation following camptocormia is the most important factor in the beneficial effect of treatments, yielding an explanation for the negative result of case \#1 patient. In contrast, lumbar spine magnetic resonance imaging of cases \#4, \#5, and \#7 patients did not show paraspinal muscle degeneration. However, it is controversial whether such fatty degeneration is a part of general aging process or a pathologic process due to Parkinson's disease.

From current literature, the pathophysiology of camptocormia is not clear, therefore, whether it is due to Parkinson's disease progression or simply occurs more frequently in individuals with progressive back muscle atrophy is questionable.

There are several limitations in this study. First, we

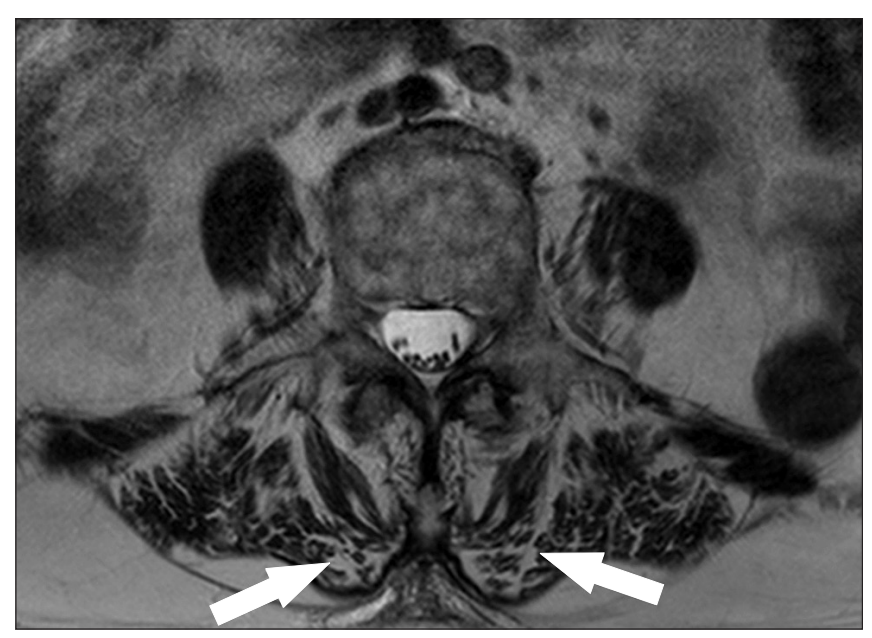

Fig. 4. Lumbar spine magnetic resonance imaging of patient \#1. The arrows indicate atrophic change of paraspinal muscle in magnetic resonance imaging T2 image of patient \#1. conducted X-ray evaluation only pre-rehabilitation. We could set other more objective points for measuring flexion angles if we conducted post-rehabilitation treatment $\mathrm{X}$-rays for all patients in exact lateral aspect. Second, comorbidities such as musculoskeletal problems and related pain that can affect gait endurance were not thoroughly evaluated.

The advantage of rehabilitation treatment in camptocormia as presented in this study is that it is cost-effective and sustainable during follow-up periods (2-26 months), when in cooperation with the patient. Finally, continuous follow-up of treatment results and possible aggravations is easier, compared to surgical treatment.

In order to verify the efficacy of rehabilitation treatment for camptocormia, objective standards for determining treatment efficacy are desperately needed. Rather than relying on the patient's subjective assessment of improvement in their symptoms, an objective standard needs to be established. Currently, visual analog scales score, gait endurance, static vertebral angle, and subjective contentment are available to assess conservative treatment results. However, problems arise due to the difficulty of defining objective standards. Some of our patients do not present signs of camptocormia before walking, but once they start walking the kyphotic angle increases (Fig. 5). Therefore, it is challenging to determine an objective time point to measure the kyphotic angle. In addition,

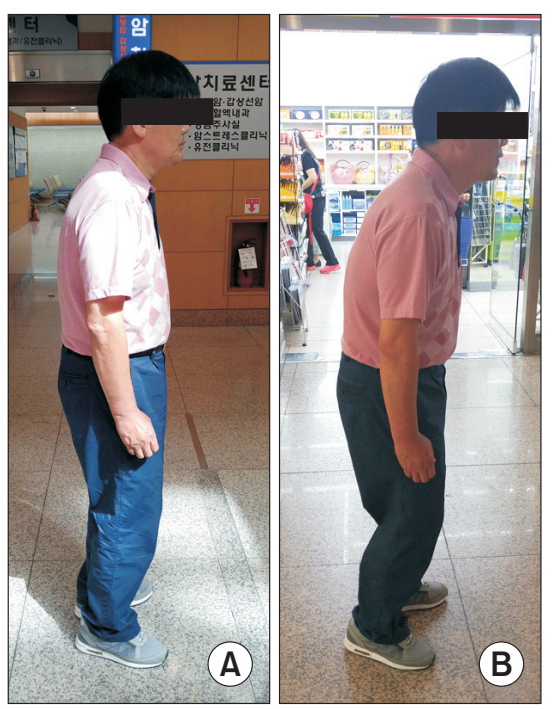

Fig. 5. Increased angle of camptocormia during ambulation of patient \#9. (A) At the beginning, angle of camptocormia is smaller. (B) After 10 minutes of gait, angle of camptocormia increased. 
gait endurance reduces as the kyphotic angle increases with walking. However, such gait endurance varies from patient to patient, and in most cases, within ten minutes of walking the kyphotic posture is so exacerbated to the extent the patient is not able to walk anymore, that practical measurement is difficult. Also in the case of static angle change, there is no definitive anatomical landmark or standards in frontal or lateral pictures such that comparative analysis can easily be influenced by the investigator's opinion $[1,21]$.

In conclusion, it is not known whether camptocormia in Parkinson's disease is a clinical characteristic of Parkinson's disease or a comorbidity due to aging. No surgical treatment protocol is available. In this study, therapeutic exercise has been tested and shown to improve ADL and motor symptoms present in Parkinson's disease with camptocormia. Therefore, we suggest that conservative treatments such as backpack-wearing or back extensor strengthening exercise should be used as the first approach, rather than invasive methods such as orthopedic surgery or bilateral subthalamic deep brain stimulation. In the future, a conservative treatment study with a larger group of patients is needed.

\section{CONFLICT OF INTEREST}

No potential conflict of interest relevant to this article was reported

\section{REFERENCES}

1. Asahi T, Taguchi Y, Hayashi N, Hamada H, Dougu N, Takashima S, et al. Bilateral subthalamic deep brain stimulation for camptocormia associated with Parkinson's disease. Stereotact Funct Neurosurg 2011;89: 173-7.

2. Seki M, Takahashi K, Koto A, Mihara B, Morita Y, Isozumi K, et al. Camptocormia in Japanese patients with Parkinson's disease: a multicenter study. Mov Disord 2011;26:2567-71.

3. Tiple D, Fabbrini G, Colosimo C, Ottaviani D, Camerota F, Defazio G, et al. Camptocormia in Parkinson disease: an epidemiological and clinical study. J Neurol Neurosurg Psychiatry 2009;80:145-8.

4. Srivanitchapoom P, Hallett M. Camptocormia in Parkinson's disease: definition, epidemiology, patho- genesis and treatment modalities. J Neurol Neurosurg Psychiatry 2016;87:75-85.

5. Azher SN, Jankovic J. Camptocormia: pathogenesis, classification, and response to therapy. Neurology 2005;65:355-9.

6. Ye BK, Kim HS, Kim YW. Correction of camptocormia using a cruciform anterior spinal hyperextension brace and back extensor strengthening exercise in a patient with Parkinson disease. Ann Rehabil Med 2015;39:128-32.

7. Colosimo C, Salvatori FM. Injection of the iliopsoas muscle with botulinum toxin in camptocormia. Mov Disord 2009;24:316-7.

8. Bloch F, Houeto JL, Tezenas du Montcel S, Bonneville F, Etchepare F, Welter ML, et al. Parkinson's disease with camptocormia. J Neurol Neurosurg Psychiatry 2006;77:1223-8.

9. Doherty KM, van de Warrenburg BP, Peralta MC, Silveira-Moriyama L, Azulay JP, Gershanik OS, et al. Postural deformities in Parkinson's disease. Lancet Neurol 2011;10:538-49.

10. von Coelln R, Raible A, Gasser T, Asmus F. Ultrasoundguided injection of the iliopsoas muscle with botulinum toxin in camptocormia. Mov Disord 2008;23:88992.

11. Yamada K, Goto S, Matsuzaki K, Tamura T, Murase $\mathrm{N}$, Shimazu H, et al. Alleviation of camptocormia by bilateral subthalamic nucleus stimulation in a patient with Parkinson's disease. Parkinsonism Relat Disord 2006;12:372-5.

12. Capelle HH, Schrader C, Blahak C, Fogel W, Kinfe TM, Baezner H, et al. Deep brain stimulation for camptocormia in dystonia and Parkinson's disease. J Neurol 2011;258:96-103.

13. Hellmann MA, Djaldetti R, Israel Z, Melamed E. Effect of deep brain subthalamic stimulation on camptocormia and postural abnormalities in idiopathic Parkinson's disease. Mov Disord 2006;21:2008-10.

14. Umemura A, Oka Y, Ohkita K, Yamawaki T, Yamada K. Effect of subthalamic deep brain stimulation on postural abnormality in Parkinson disease. J Neurosurg 2010;112:1283-8.

15. Thani NB, Bala A, Kimber TE, Lind CR. High-frequency pallidal stimulation for camptocormia in Parkinson disease: case report. Neurosurgery 2011;68:E1501-5.

16. Sako W, Nishio M, Maruo T, Shimazu H, Matsuzaki K, 
Tamura T, et al. Subthalamic nucleus deep brain stimulation for camptocormia associated with Parkinson's disease. Mov Disord 2009;24:1076-9.

17. Upadhyaya CD, Starr PA, Mummaneni PV. Spinal deformity and Parkinson disease: a treatment algorithm. Neurosurg Focus 2010;28:E5.

18. Gerton BK, Theeler B, Samii A. Backpack treatment for camptocormia. Mov Disord 2010;25:247-8.

19. Schroeteler FE, Fietzek UM, Ziegler K, Ceballos-Baumann AO. Upright posture in parkinsonian camptocormia using a high-frame walker with forearm support. Mov Disord 2011;26:1560-1.
20. de Seze MP, Creuze A, de Seze M, Mazaux JM. An orthosis and physiotherapy programme for camptocormia: a prospective case study. J Rehabil Med 2008;40: 761-765.

21. Capecci M, Serpicelli C, Fiorentini L, Censi G, Ferretti M, Orni C, et al. Postural rehabilitation and Kinesio taping for axial postural disorders in Parkinson's disease. Arch Phys Med Rehabil 2014;95:1067-75.

22. Schulz-Schaeffer WJ, Margraf NG, Munser S, Wrede A, Buhmann C, Deuschl G, et al. Effect of neurostimulation on camptocormia in Parkinson's disease depends on symptom duration. Mov Disord 2015;30:368-72. 